\title{
TU/e ENHOUEN

\section{An eigencurrent approach to the analysis of electrically large 3-D structures using linear embedding via Green's operators}

\section{Citation for published version (APA):}

Lancellotti, V., Hon, de, B. P., \& Tijhuis, A. G. (2009). An eigencurrent approach to the analysis of electrically large 3-D structures using linear embedding via Green's operators. IEEE Transactions on Antennas and Propagation, 57(11), 3575-3585. https://doi.org/10.1109/TAP.2009.2027616

DOI:

10.1109/TAP.2009.2027616

Document status and date:

Published: 01/01/2009

\section{Document Version:}

Publisher's PDF, also known as Version of Record (includes final page, issue and volume numbers)

\section{Please check the document version of this publication:}

- A submitted manuscript is the version of the article upon submission and before peer-review. There can be important differences between the submitted version and the official published version of record. People interested in the research are advised to contact the author for the final version of the publication, or visit the $\mathrm{DOI}$ to the publisher's website.

- The final author version and the galley proof are versions of the publication after peer review.

- The final published version features the final layout of the paper including the volume, issue and page numbers.

Link to publication

\section{General rights}

Copyright and moral rights for the publications made accessible in the public portal are retained by the authors and/or other copyright owners and it is a condition of accessing publications that users recognise and abide by the legal requirements associated with these rights.

- Users may download and print one copy of any publication from the public portal for the purpose of private study or research.

- You may not further distribute the material or use it for any profit-making activity or commercial gain

- You may freely distribute the URL identifying the publication in the public portal.

If the publication is distributed under the terms of Article 25fa of the Dutch Copyright Act, indicated by the "Taverne" license above, please follow below link for the End User Agreement:

www.tue.nl/taverne

Take down policy

If you believe that this document breaches copyright please contact us at:

openaccess@tue.nl

providing details and we will investigate your claim. 


\title{
An Eigencurrent Approach to the Analysis of Electrically Large 3-D Structures Using Linear Embedding via Green's Operators
}

\author{
Vito Lancellotti, Member, IEEE, Bastiaan P. de Hon, and Anton G. Tijhuis, Member, IEEE
}

\begin{abstract}
We present an extension of the linear embedding via Green's operators (LEGO) procedure for efficiently dealing with 3-D electromagnetic composite structures. In LEGO's notion, we enclose the objects forming a structure within arbitrarily shaped domains (bricks), which (by invoking the equivalence principle) we characterize through scattering operators. In the 2-D instance, we then combined the bricks numerically, in a cascade of successive embedding steps, to build increasingly larger domains and obtain the scattering operator of the whole aggregate of objects. In the 3-D case, however, this process becomes quite soon impracticable, in that the resulting scattering matrices are too big to be stored and handled on most computers. To circumvent this hurdle, we propose a novel formulation of the electromagnetic problem based on an integral equation involving the total inverse scattering operator of the structure, which can be written analytically in terms of scattering operators of the bricks and transfer operators among them. We then solve this equation by the method of moments combined with the eigencurrent expansion method, which allows for a considerable reduction in size of the system matrix and thereby enables us to study very large structures.
\end{abstract}

Index Terms-Boundary integral equations, composite structures, diakoptics, domain decomposition method, eigencurrent expansion method, equivalence principle, method of moments (MoM).

\section{MOTIVATION AND BACKGROUND}

$\mathbf{T}$ HE scattering and radiation of electromagnetic (EM) waves from electrically large structures-such as EM band gap structures, frequency selective surfaces and antenna arrays, to name but a few examples-constitutes a problem of great practical interest. When a numerical solution is attempted (whether in the spatial or in the spectral domain) with the finite element method (FEM) [1] or the method of moments (MoM) [2], one is sooner or later faced with the issue of filling, storing and (formally) inverting a huge matrix. This may be a challenging or even unfeasible task due to both too long computational time and practically limited memory resources.

To get around these hurdles, we have developed a modular EM modelling approach, named linear embedding via Green's

Manuscript received April 09, 2009; revised July 05, 2009. First published July 14, 2009; current version published November 04, 2009. This work was supported by the post-doc fund under TU/e project no. 36/363450, and is performed in the framework of the MEMPHIS project.

The authors are with the Department of Electrical Engineering, Eindhoven University of Technology, 5600 MB Eindhoven, The Netherlands (e-mail: v.lancellotti@tue.nl; b.p.d.hon@tue.nl; a.g.tijhuis@tue.nl).

Color versions of one or more of the figures in this paper are available online at http://ieeexplore.ieee.org.

Digital Object Identifier 10.1109/TAP.2009.2027616 operators (LEGO), which relies on the decomposition of a large structure into its constituent parts [3]-[5]. Admittedly, the idea of subdividing large systems into interacting multiport subsystems, which is called diakoptics, dates back at least to the fifties [6]. In LEGO we embed an object (or many objects as well) within a "brick", which we then characterize electromagnetically by means of a scattering operator. Hence, we can say the LEGO method is a specific variety of diakoptics, in that a complicated structure is modelled by many interacting bricks which can be regarded as multiport systems with a continuous distribution of ports. In this respect, the scattering operator of a LEGO brick extends the usual notion of scattering matrix for a multiport device, where the ports may be either actual terminals [7] or just abstract terminals corresponding to the spectrum of propagating and evanescent modes in, e.g., a cylindrical waveguide [8], or a stratified medium [9].

The primary goal of LEGO is not to propose a new fast algorithm for solving boundary integral equations (BIE) (even though reduction of the overall computational time shows up as a quite welcome side effect), but rather to provide an effective procedure which makes the solution of large scale problems feasible. Nevertheless, LEGO shares the very idea of tearing apart a large structure into pieces with other approaches, such as the popular fast multipole method (FMM) [10] and the more recent nested equivalence principle algorithm (NEPAL) [11] and the equivalence principle algorithm (EPA) [12]. The FMM aims at accelerating the iterative solution of a BIE, in conjunction with any suitable numerical method, upon reducing the number of matrix-vector multiplications. To this end, the FMM relies on the subdivision of the basis functions (used to expand the unknown) into localized groups. Conversely, in LEGO the emphasis is on parts of the structure and the subdivision is done prior to any discretization [5]. In the NEPAL procedure, a large scattering problem is addressed by solving a succession of smaller problems, which are defined upon repeatedly applying Huygens' equivalence principle (EP) [13]. By contrast, in LEGO, we first describe the bricks forming a structure independently of one another, by means of their scattering operators, and then we formulate the whole original problem. Similarly to LEGO, in the EPA scheme [12] scattering operators are defined on the boundaries of domains-which enclose complicated structures with fine details-in order to reduce the number of unknowns and hence the overall computational load for scattering problems.

In the 2-D instance of LEGO [3], indeed, we did obtain the total scattering matrix of a structure numerically with a cascade of successive embedding steps. This strategy is not generally 
viable in 3-D problems, for the size of the matrix doubles at each step and a naïve application of the process would soon drain the memory of most computers, thus limiting the number of bricks that can be handled at the same time [14].

For this reason, we propose a novel approach based on the total inverse scattering operator, $\mathrm{S}^{-1}$, of the whole structure. Since $\mathrm{S}^{-1}$ can be written analytically in a formal fashion, as we will show, we use it to formulate the scattering problem through an integral equation for the whole structure. However, if we were to try solving the latter equation directly with the MoM, then in general we would fail, for the resulting system matrix can usually not be stored nor inverted, except for small problems. Therefore, we solve the relevant equation by the eigencurrent expansion method (EEM) [15], [16, Ch. 5], which is the other important idea of this work. While referring the reader to Section IV for the necessary details, here we mention that the EEM is essentially the MoM applied with a suitable set of basis and test functions, whose introduction allows us to reduce the size of the matrix to be stored and inverted. A substantive increase in speed of the overall process stems from drastically reducing the time needed to virtually fill the total inverse scattering matrix $[S]^{-1}$, as discussed in Section IV-C.

Among the distinctive features that make LEGO particularly efficient, we mention:

- LEGO bricks may be arbitrarily combined and re-used to form new structures, without having to recompute the relevant scattering operators;

- the interaction between the bricks is mediated by transfer operators that depend only on the shape and relative position of the bricks (not on their content), thus they may be computed once for a given spatial distribution;

- LEGO inherently lends itself to parallel computing, since the calculation of the transfer matrices involves just pairs of bricks at a time;

- local optimization of a large structure can efficiently be carried out by identifying a designated brick in which the EM properties are supposed to vary, whilst leaving the other bricks unchanged.

The rest of this paper is organized as follows. In Section II we outline the notion of LEGO brick and introduce its scattering operator, whereas in Section III we show how to formulate the EM problem of a composite structure, represented with many bricks, through an integral equation involving $S^{-1}$. In Section IV we outline the solution of the latter equation with the MoM and the EEM. Finally, in Section V we discuss the validation of the code implementing LEGO, the convergence of the EEM and the time requirements of the overall approach.

\section{Definition of 3-D LEGO EM BRICKS AND THEIR SCATTERING OPERATORS}

In the LEGO concept, we start solving the EM problem by separating a large complex structure into basic constituents, which may actually consist of one or more objects with arbitrary shape. At this stage, the underlying goal is to provide a full-wave description of each part that is independent of all the others as well as of the external sources. To this end, we consider a (homogeneous) background medium, in which the whole structure is embedded, and carve out $N_{D}$ bounded

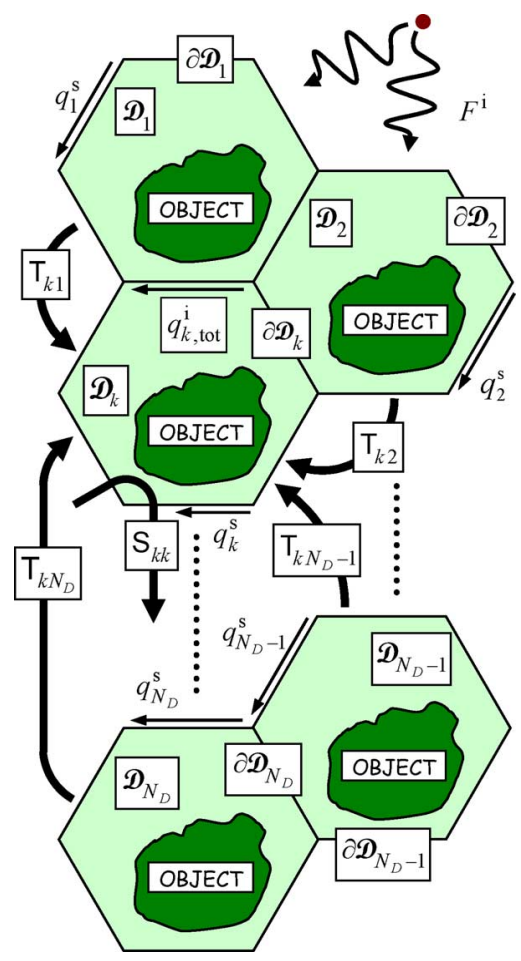

Fig. 1. Modified LEGO concept: a large composite structure is decomposed in 3-D LEGO EM bricks (see Fig. 2) each endowed with its own scattering operator $\mathrm{S}_{k k}(11)$; then the multiple scattering occurring among the bricks is described by transfer operators $\mathrm{T}_{k n}$ (13) and finally captured in the total inverse scattering operator $S^{-1}(17)$.

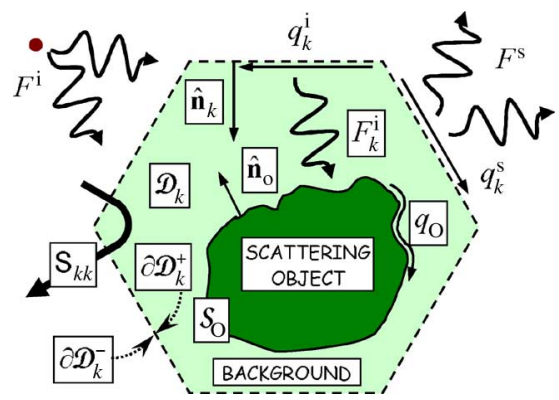

Fig. 2. A 3-D LEGO EM brick consists of a bounded volume $\mathcal{D}_{k}$ (carved out of a background homogeneous medium) enclosing a scattering object. The EM behavior of the brick - which depends on the nature of the enclosed scattering object-is fully described by its scattering operator $S_{k k}$.

domains $\mathcal{D}_{k}, k=1, \ldots, N_{D}$, each enclosing a body, as exemplified in Figs. 1 and 2. Apparently, we are at liberty of choosing any shape for the boundary $\partial \mathcal{D}_{k}$ of $\mathcal{D}_{k}$-which adds great flexibility to LEGO. Nonetheless a certain degree of symmetry is expedient, for this eventually facilitates the process of stacking many such domains (in the three spatial directions) in order to characterize the original complete structure. We refer to the domains $\mathcal{D}_{k}$ as LEGO EM bricks [4] and we characterize their EM behavior by means of scattering operators $S_{k k}$.

To introduce and derive $S_{k k}$ let us consider the EM problem outlined in Fig. 2, consisting of just a single brick $\mathcal{D}_{k}$, i.e. without all the other bricks comprising the structure, and arbitrary sources outside $\mathcal{D}_{k}$. The brick $\mathcal{D}_{k}$ embeds an object which, being illuminated by an incident field $F^{\mathrm{i}}$, develops and 
TABLE I

PROPAGATORS USED IN LEGO

Propagators are $2 \times 2$ matrices whose entries are rank- 2 dyadic integrodifferential operators involving the scalar Green's function $G(R)$ of the background medium. $\kappa_{0}$ denotes the vacuum wavenumber. The unit normal to $\partial \mathcal{D}_{k}$ points always inward $\mathcal{D}_{k}$ (Fig. 2).

NOMENCLATURE

$G(R)=\exp (-\mathrm{j} \kappa R) /(4 \pi R), \quad R=\left|\boldsymbol{r}-\boldsymbol{r}^{\prime}\right|, \quad \kappa=\kappa_{0} \sqrt{\varepsilon \mu}$

$\underline{\underline{I}}_{\mathrm{s}}=\underline{\underline{I}}-\hat{\boldsymbol{n}} \hat{\boldsymbol{n}}, \quad \nabla_{\mathrm{s}}=\underline{\underline{I}}_{\mathrm{s}} \cdot \nabla=\nabla-\hat{\boldsymbol{n}}(\hat{\boldsymbol{n}} \cdot \nabla), \quad \nabla_{\mathrm{s}}^{\prime}=-\nabla_{\mathrm{s}}$

$\partial \mathcal{D}_{k}^{+} \rightarrow \partial \mathcal{D}_{k}^{+}$(from incident currents to incident fields), $\boldsymbol{r} \in \partial \mathcal{D}_{k}^{+}$

$\left(\mathrm{P}_{k k}^{\mathrm{i}}\right)_{11}=-\mathrm{j} \int_{\partial \mathcal{D}_{k}^{+}} \mathrm{d}^{2} r^{\prime}\left[\kappa G(R) \underline{\underline{I}}_{\mathrm{S}}+\frac{1}{\kappa} \nabla_{\mathrm{s}} G(R) \nabla_{\mathrm{s}}^{\prime}\right]$.

$\left(\mathrm{P}_{k k}^{\mathrm{i}}\right)_{22}=-\left(\mathrm{P}_{k k}^{\mathrm{i}}\right)_{11}$

$\left(\mathrm{P}_{k k}^{\mathrm{i}}\right)_{12}=-$ P.V. $\int_{\partial \mathcal{D}_{k}^{+}} \mathrm{d}^{2} r^{\prime} \nabla_{\mathrm{S}}^{\prime} G(R) \times \underline{\underline{I}}_{\mathrm{S}} \cdot+\frac{1}{2} \hat{\boldsymbol{n}}_{k} \times \underline{\underline{I}}_{\mathrm{S}}$.

$\left(\mathrm{P}_{k k}^{\mathrm{i}}\right)_{21}=$ P.V. $\int_{\partial \mathcal{D}_{k}^{+}} \mathrm{d}^{2} r^{\prime} \nabla_{\mathrm{s}} G(R) \times \underline{\underline{I}}_{\mathrm{S}} \cdot-\frac{1}{2} \hat{\boldsymbol{n}}_{k} \times \underline{\underline{I}}_{\mathrm{s}}$.

$\partial \mathcal{D}_{k}^{-} \rightarrow \partial \mathcal{D}_{k}^{-}$(from scattered currents to scattered fields), $\boldsymbol{r} \in \partial \mathcal{D}_{k}^{-}$

$\left(\mathrm{P}_{k k}^{\mathrm{S}}\right)_{11}=-\mathrm{j} \int_{\partial \mathcal{D}_{k}^{-}} \mathrm{d}^{2} r^{\prime}\left[\kappa G(R) \underline{I}_{\mathrm{S}}+\frac{1}{\kappa} \nabla_{\mathrm{s}} G(R) \nabla_{\mathrm{S}}^{\prime}\right]$.

$\left(\mathrm{P}_{k k}^{\mathrm{s}}\right)_{22}=-\left(\mathrm{P}_{k k}^{\mathrm{s}}\right)_{11}$

$\left(\mathrm{P}_{k k}^{\mathrm{s}}\right)_{12}=-$ P.V. $\int_{\partial \mathcal{D}_{k}^{-}} \mathrm{d}^{2} r^{\prime} \nabla_{\mathrm{S}}^{\prime} G(R) \times \underline{\underline{I}} \cdot \mathrm{s} \cdot-\frac{1}{2} \hat{\boldsymbol{n}}_{k} \times \underline{\underline{I}} \mathrm{~s}$.

$\left(\mathrm{P}_{k k}^{\mathrm{s}}\right)_{21}=$ P.V. $\int_{\partial \mathcal{D}_{k}^{-}} \mathrm{d}^{2} r^{\prime} \nabla_{\mathrm{s}} G(R) \times \underline{\underline{I}}_{\mathrm{s}} \cdot+\frac{1}{2} \hat{\boldsymbol{n}}_{k} \times \underline{\underline{I}}_{\mathrm{S}}$.

$\partial \mathcal{D}_{n}^{-} \rightarrow \partial \mathcal{D}_{k}^{+}, n \neq k$

(from scattered currents to incident fields), $r \in \partial \mathcal{D}_{k}^{+}$

$\left(\mathrm{P}_{k n}\right)_{11}=\left(\mathrm{P}_{k n}\right)_{12}=0$

$\left(\mathrm{P}_{k n}\right)_{21}=$ P.V. $\int_{\partial \mathcal{D}_{n}^{-}} \mathrm{d}^{2} r^{\prime} \nabla_{\mathrm{S}} G(R) \times \underline{\underline{I}}_{\mathrm{s}} \cdot+\frac{1}{2} \hat{\boldsymbol{n}}_{n} \times \underline{\underline{I}}_{\mathrm{s}} \cdot$

$\left(\mathrm{P}_{k n}\right)_{22}=\mathrm{j} \int_{\partial \mathcal{D}_{n}^{-}} \mathrm{d}^{2} r^{\prime}\left[\kappa G(R) \underline{I}_{\mathrm{s}}+\frac{1}{\kappa} \nabla_{\mathrm{S}} G(R) \nabla_{\mathrm{S}}^{\prime}\right]$.

sustains induced (secondary) sources that radiate the scattered field $F^{\mathrm{s}}$, where

$$
F^{\mathrm{i}}=\left[\begin{array}{c}
\boldsymbol{E}^{\mathrm{i}} / \sqrt{\eta} \\
\sqrt{\eta} \boldsymbol{H}^{\mathrm{i}}
\end{array}\right], \quad F^{\mathrm{s}}=\left[\begin{array}{c}
\boldsymbol{E}^{\mathrm{s}} / \sqrt{\eta} \\
\sqrt{\eta} \boldsymbol{H}^{\mathrm{s}}
\end{array}\right]
$$

and $\eta=\sqrt{\mu / \varepsilon}$ is the intrinsic impedance of the background medium. We assume a time-harmonic, $\exp (\mathrm{j} \omega t)$, dependence for fields and currents throughout.

From the standpoint of an observer inside $\mathcal{D}_{k}$, the EM problem looks the same if we replace the actual sources with equivalent current densities $q_{k}^{\mathrm{i}}$ on $\partial \mathcal{D}_{k}^{+}$that reproduce the incident field $F^{\mathrm{i}}$. Conversely, an observer sitting outside $\mathcal{D}_{k}$ detects the same scattered field $F^{\mathrm{s}}$, provided we place proper equivalent current densities $q_{k}^{\mathrm{s}}$ on $\partial \mathcal{D}_{k}^{-}$, set against the background medium. The quantities $q_{k}^{\mathrm{i}}$ and $q_{k}^{\mathrm{s}}$ are defined as

$$
q_{k}^{\mathrm{i}}=\left[\begin{array}{c}
\sqrt{\eta} \boldsymbol{J}_{k}^{\mathrm{i}} \\
-\boldsymbol{M}_{k}^{\mathrm{i}} / \sqrt{\eta}
\end{array}\right], \quad q_{k}^{\mathrm{s}}=\left[\begin{array}{c}
\sqrt{\eta} \boldsymbol{J}_{k}^{\mathrm{s}} \\
-\boldsymbol{M}_{k}^{\mathrm{s}} / \sqrt{\eta}
\end{array}\right]
$$

where $\boldsymbol{J}$ and $\boldsymbol{M}$ denote electric and magnetic current densities. In (1) and (2) $\eta$ serves not only as a mere normalization factor-so $F_{k}^{\mathrm{s}, \mathrm{i}}$ and $q_{k}^{\mathrm{s}, \mathrm{i}}$ possess the physical dimension of a power wave per unit length-but also for the purpose of balancing the matrices that arise in the MoM solution of the BIEs
TABLE II

EXPLICIT FORM OF (8) IN CASES OF INTEREST

The subscript " $b$ " denotes operators and quantities within the medium comprising the penetrable body. $\eta_{0}\left(\kappa_{0}\right)$ is the intrinsic impedance (wavenumber) of vacuum. The unit normal to $\mathcal{S}_{0}$ points inward $\mathcal{D}_{k}$. NOMENCLATURE

$G(R)=\exp (-\mathrm{j} \kappa R) /(4 \pi R), \quad G_{\mathrm{b}}(R)=\exp \left(-\mathrm{j} \kappa_{\mathrm{b}} R\right) /(4 \pi R)$

$\kappa=\kappa_{0} \sqrt{\varepsilon \mu}, \quad \eta=\eta_{0} \sqrt{\mu / \varepsilon}, \quad \kappa_{\mathrm{b}}=\kappa_{0} \sqrt{\varepsilon_{\mathrm{b}} \mu_{\mathrm{b}}}, \quad \eta_{\mathrm{b}}=\eta_{0} \sqrt{\mu_{\mathrm{b}} / \varepsilon_{\mathrm{b}}}$

$R=\left|\boldsymbol{r}-\boldsymbol{r}^{\prime}\right|, \quad \underline{\underline{I}}=\underline{\underline{\mathrm{I}}}-\hat{\boldsymbol{n}}_{\mathrm{o}} \hat{\boldsymbol{n}}_{\mathrm{O}}, \quad \nabla_{\mathrm{S}}=\underline{I}_{\mathrm{S}} \cdot \nabla, \quad \nabla_{\mathrm{S}}^{\prime}=-\nabla_{\mathrm{S}}$

BASIC RANK-2 DYADIC OPERATORS

$\mathcal{L}=-\mathrm{j} \int_{\mathcal{S}_{\mathrm{O}}} \mathrm{d}^{2} r^{\prime}\left[\kappa G(R) \underline{\underline{I}}{ }_{\mathrm{S}}+\frac{1}{\kappa} \nabla_{\mathrm{S}} G(R) \nabla_{\mathrm{S}}^{\prime}\right] \cdot, \quad \boldsymbol{r} \in \mathcal{S}_{\mathrm{O}}$

$\mathcal{K}^{ \pm}=$P.V. $\int_{\mathcal{S}_{\mathrm{O}}} \mathrm{d}^{2} r^{\prime} \nabla_{\mathrm{S}}^{\prime} G(R) \times \underline{\underline{I}}_{\mathrm{S}} \cdot \pm \frac{1}{2} \hat{\boldsymbol{n}}_{\mathrm{O}} \times \underline{\underline{I}}_{\mathrm{s}} \cdot, \quad \boldsymbol{r} \in \mathcal{S}_{\mathrm{O}}$

$\mathcal{L}_{\mathrm{b}}=-\mathrm{j} \int_{\mathcal{S}_{\mathrm{O}}} \mathrm{d}^{2} r^{\prime}\left[\kappa_{\mathrm{b}} G_{\mathrm{b}}(R) \underline{\underline{I}}_{\mathrm{s}}+\frac{1}{\kappa_{\mathrm{b}}} \nabla_{\mathrm{s}} G_{\mathrm{b}}(R) \nabla_{\mathrm{s}}^{\prime}\right], \quad \boldsymbol{r} \in \mathcal{S}_{\mathrm{O}}$

$\mathcal{K}_{\mathrm{b}}^{ \pm}=$P.V. $\int_{\mathcal{S}_{\mathrm{O}}} \mathrm{d}^{2} r^{\prime} \nabla_{\mathrm{s}}^{\prime} G_{\mathrm{b}}(R) \times \underline{\underline{I}}_{\mathrm{S}} \cdot \pm \frac{1}{2} \hat{\boldsymbol{n}}_{\mathrm{O}} \times \underline{\underline{I}}_{\mathrm{S}} \cdot, \quad \boldsymbol{r} \in \mathcal{S}_{\mathrm{O}}$

Perfect Electric Conductor

MFIE $(\alpha=0)$, CFIE $(0<\alpha<1)$, EFIE $(\alpha=1)$

$q_{\mathrm{o}}=\left[\begin{array}{c}\sqrt{\eta} \boldsymbol{J}_{\mathrm{o}} \\ 0\end{array}\right], \quad F_{\mathrm{to}}^{\mathrm{i}}=\left[\begin{array}{c}\alpha \boldsymbol{E}_{\mathrm{to}}^{\mathrm{i}} / \sqrt{\eta}+(1-\alpha) \sqrt{\eta} \boldsymbol{H}_{\mathrm{to}}^{\mathrm{i}} \\ 0\end{array}\right]$

$\mathrm{X}_{\mathrm{oo}}=\left[\begin{array}{cc}\alpha \mathcal{L}+(1-\alpha) \mathcal{K}^{+} & 0 \\ 0 & 0\end{array}\right]$

Penetrable body:

$q_{\mathrm{o}}=\left[\begin{array}{c}\sqrt{\eta} \boldsymbol{J}_{\mathrm{O}} \\ -\boldsymbol{M}_{\mathrm{o}} / \sqrt{\eta}\end{array}\right], \quad F_{\mathrm{to}}^{\mathrm{i}}=\left[\begin{array}{c}\boldsymbol{E}_{\mathrm{to}}^{\mathrm{i}} / \sqrt{\eta} \\ \sqrt{\eta} \boldsymbol{H}_{\mathrm{to}}^{\mathrm{i}}\end{array}\right]$

PMCHW equations

$\mathrm{X}_{\mathrm{oo}}=\left[\begin{array}{cc}\mathcal{L}+\left(\eta_{\mathrm{b}} / \eta\right) \mathcal{L}_{\mathrm{b}} & -\mathcal{K}^{+}-\mathcal{K}_{\mathrm{b}}^{-} \\ -\mathcal{K}^{+}-\mathcal{K}_{\mathrm{b}}^{-} & -\mathcal{L}-\left(\eta / \eta_{\mathrm{b}}\right) \mathcal{L}_{\mathrm{b}}\end{array}\right]$

Müller equations

$\mathrm{X}_{\mathrm{oo}}=\left[\begin{array}{cc}\mathcal{L}-\eta_{\mathrm{b}} \varepsilon_{\mathrm{b}} \mathcal{L}_{\mathrm{b}} /(\eta \varepsilon) & -\mathcal{K}^{-}+\left(\varepsilon_{\mathrm{b}} / \varepsilon\right) \mathcal{K}_{\mathrm{b}}^{+} \\ -\mathcal{K}^{-}+\left(\mu_{\mathrm{b}} / \mu\right) \mathcal{K}_{\mathrm{b}}^{+} & -\mathcal{L}+\eta \mu_{\mathrm{b}} \mathcal{L}_{\mathrm{b}} /\left(\eta_{\mathrm{b}} \mu\right)\end{array}\right]$

involved in the numerical derivation of the scattering operator (see definition of operators in Tables I-III).

The equivalence principle (in the formalism of Love, [13], [17], [18]), which we have tacitly invoked twice in our reasoning, prescribes that the equivalent current densities be related to the tangential fields on $\partial \mathcal{D}_{k}$ via

$$
q_{k}^{\mathrm{i}}=\left[\begin{array}{c}
\sqrt{\eta} \hat{\boldsymbol{n}}_{k} \times \boldsymbol{H}_{k}^{\mathrm{i}} \\
\hat{\boldsymbol{n}}_{k} \times \boldsymbol{E}_{k}^{\mathrm{i}} / \sqrt{\eta}
\end{array}\right], \quad q_{k}^{\mathrm{s}}=\left[\begin{array}{c}
\sqrt{\eta} \boldsymbol{H}_{k}^{\mathrm{s}} \times \hat{\boldsymbol{n}}_{k} \\
\boldsymbol{E}_{k}^{\mathrm{s}} \times \hat{\boldsymbol{n}}_{k} / \sqrt{\eta}
\end{array}\right]
$$

which indeed provides a mean to compute them. However, we prefer to deduce $q_{k}^{\mathrm{s}, \mathrm{i}}$ through two BIEs posed on either sides of $\partial \mathcal{D}_{k}$ and entailing the so called propagators, namely

$$
\begin{aligned}
& \mathrm{P}_{k k}^{\mathrm{i}} q_{k}^{\mathrm{i}}=\int_{\partial \mathcal{D}_{k}^{+}} \mathrm{d}^{2} r^{\prime} P_{k k}^{\mathrm{i}}\left(\boldsymbol{r}, \boldsymbol{r}^{\prime}\right) q_{k}^{\mathrm{i}}\left(\boldsymbol{r}^{\prime}\right)=F_{\mathrm{t} k}^{\mathrm{i}}, \quad \boldsymbol{r} \in \partial \mathcal{D}_{k}^{+} \\
& \mathrm{P}_{k k}^{\mathrm{s}} q_{k}^{\mathrm{s}}=\int_{\partial \mathcal{D}_{k}^{-}} \mathrm{d}^{2} r^{\prime} P_{k k}^{\mathrm{s}}\left(\boldsymbol{r}, \boldsymbol{r}^{\prime}\right) q_{k}^{\mathrm{s}}\left(\boldsymbol{r}^{\prime}\right)=F_{\mathrm{t} k}^{\mathrm{s}}, \quad \boldsymbol{r} \in \partial \mathcal{D}_{k}^{-}
\end{aligned}
$$

inasmuch as they lead to a stabler numerical scheme than the combination of (2) and (3) does. In (4) and (5), integration is 
TABLE III

PRopagators USED IN LEGO (CONTINUED)

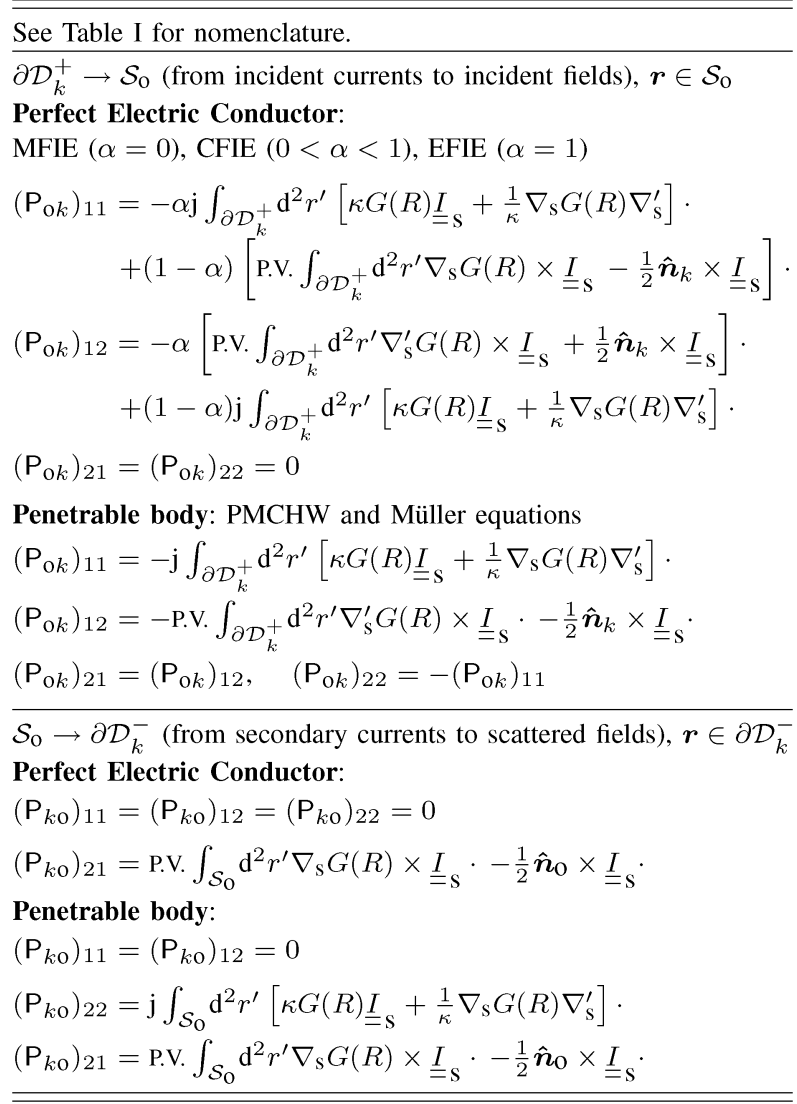

carried out over a brick's boundary and $\mathrm{d}^{2} r^{\prime}$ denotes the area element. The operators $\mathrm{P}_{k k}^{\mathrm{i}}$ and $\mathrm{P}_{k k}^{\mathrm{s}}$ are explicitly given in Table I, whilst the forcing terms are

$$
F_{\mathrm{t} k}^{\mathrm{i}}=\left[\begin{array}{c}
0 \\
\sqrt{\eta} \boldsymbol{H}_{\mathrm{t} k}^{\mathrm{i}}
\end{array}\right], \quad F_{\mathrm{t} k}^{\mathrm{s}}=\left[\begin{array}{c}
0 \\
\sqrt{\eta} \boldsymbol{H}_{\mathrm{t} k}^{\mathrm{s}}
\end{array}\right]
$$

i.e., they involve only the tangential magnetic field components. In words, (4) requires that the fields generated by $q_{k}^{\mathrm{i}}$ inside $\mathcal{D}_{k}$ equate the true incident fields on $\partial \mathcal{D}_{k}^{+}$; similar arguments apply to (5). We refer to the operators in (4) and (5) as propagators, since in general they relate currents on one brick's boundary to fields on another brick's; in this special instance, though, fields and sources clearly share the same surface. It is worth noticing that the numerical solution of (4) and (5)—with the MoM and a symmetric inner product [19]—yields symmetric propagation matrices $\left[P_{k k}^{\mathrm{i}, \mathrm{s}}\right]$.

We now introduce the (dimensionless) scattering operators $\mathrm{S}_{k k}$ through

$$
q_{k}^{\mathrm{s}}=\mathrm{S}_{k k} q_{k}^{\mathrm{i}}=\int_{\partial \mathcal{D}_{k}^{+}} \mathrm{d}^{2} r^{\prime} S_{k k}\left(\boldsymbol{r}, \boldsymbol{r}^{\prime}\right) q_{k}^{\mathrm{i}}\left(\boldsymbol{r}^{\prime}\right), \quad \boldsymbol{r} \in \partial \mathcal{D}_{k}^{-}
$$

that is, a mapping between incident and scattered equivalent current densities on either side of $\partial \mathcal{D}_{k}$.

To find an operative expression for $S_{k k}$, we first solve for the secondary sources, say $q_{\mathrm{o}}$, induced over the object inside $\mathcal{D}_{k}$. Then, we relate the scattered fields on $\partial \mathcal{D}_{k}^{-}$to $q_{\mathrm{o}}$. Generally, the calculation of $q_{\mathrm{o}}$ may entail posing a set of coupled BIEs on the object's boundary $\mathcal{S}_{\mathrm{o}}$, viz.,

$$
\mathrm{X}_{\mathrm{oo}} q_{\mathrm{o}}=-F_{\text {to }}^{\mathrm{i}}, \text { on } \mathcal{S}_{\mathrm{o}}
$$

where the actual form of $\mathrm{X}_{\mathrm{oo}}, q_{\mathrm{o}}$ and $F_{\text {to }}^{\mathrm{i}}$ depends on both the object's nature and the adopted formulation. In Table II we have collected the relevant expressions of $\mathrm{X}_{\mathrm{oo}}, q_{\mathrm{o}}$ and $F_{\text {to }}^{\mathrm{i}}$ for the notable instances of a perfectly electrically conducting (PEC) object (formulated with the EFIE [19], MFIE [19], CFIE [20]), and a penetrable body (formulated with the PMCHW [19] and Müller [21] equations).

It is important to note that in (8) the forcing term consists of the fields radiated by the equivalent incident currents $q_{k}^{\mathrm{i}}$ instead of the actual external sources. In fact, we obtain $F_{\text {to }}^{\mathrm{i}}$ by acting on $q_{k}^{\mathrm{i}}$ with the propagator from $\partial \mathcal{D}_{k}^{+}$to $\mathcal{S}_{\mathrm{o}}$, viz.,

$$
F_{\text {to }}^{\mathrm{i}}=\mathrm{P}_{\mathrm{o} k} q_{k}^{\mathrm{i}}=\int_{\partial \mathcal{D}_{k}^{+}} \mathrm{d}^{2} r^{\prime} P_{\mathrm{o} k}\left(\boldsymbol{r}, \boldsymbol{r}^{\prime}\right) q_{k}^{\mathrm{i}}\left(\boldsymbol{r}^{\prime}\right), \quad \boldsymbol{r} \in \mathcal{S}_{\mathrm{o}} .
$$

Similarly, we derive $F_{\mathrm{t} k}^{\mathrm{s}}$ from $q_{\mathrm{o}}$ by applying a propagator from $\mathcal{S}_{\mathrm{o}}$ to $\partial \mathcal{D}_{k}^{-}$, namely,

$$
F_{\mathrm{t} k}^{\mathrm{s}}=\mathrm{P}_{k \mathrm{o}} q_{\mathrm{o}}=\int_{\mathcal{S}_{\mathrm{o}}} \mathrm{d}^{2} r^{\prime} P_{k \mathrm{o}}\left(\boldsymbol{r}, \boldsymbol{r}^{\prime}\right) q_{\mathrm{o}}\left(\boldsymbol{r}^{\prime}\right), \quad \boldsymbol{r} \in \partial \mathcal{D}_{k}^{-} .
$$

For the sake of completeness, in Table III we list the expressions of $\mathrm{P}_{\mathrm{o} k}$ and $\mathrm{P}_{k o}$ pertinent to the cases of PEC and penetrable objects.

Eventually, upon eliminating $q_{\mathrm{o}}$ and the fields from (5), (8), (9) and (10), we arrive at the following expression for the scattering operators

$$
\mathrm{S}_{k k}=-\left(\mathrm{P}_{k k}^{\mathrm{s}}\right)^{-1} \mathrm{P}_{k \mathrm{o}}\left(\mathrm{X}_{\mathrm{oo}}\right)^{-1} \mathrm{P}_{\mathrm{o} k}
$$

which provides us with a suitable recipe to compute $S_{k k}$ numerically by the MoM. We notice that, as required, the $S_{k k}$ do not depend on one another nor on the external sources. In fact, (4), which we have not used to derive (11), allows us to compute the incident currents $q_{k}^{\mathrm{i}}$ from $F_{\mathrm{t} k}^{\mathrm{i}}$. In the trivial instance of just one brick, then plugging $q_{k}^{\mathrm{i}}$ into (7) yields the scattered currents. More generally, although $\mathrm{S}_{k k}$ is all we need to fully characterize a LEGO brick, we need many of them to describe a composite structure: how the bricks combine in a EM sense will be the subject of the next section.

\section{FORMULATION THROUGH THE TOTAL INVERSE SCATTERING OPERATOR $\mathrm{S}^{-1}$}

Unlike the strategy adopted in the 2-D LEGO method [3], where in subsequent steps we (numerically) built a total scattering operator by combining pairs of increasingly larger bricks, here we take another route. To be specific, we analytically derive the total inverse scattering operator $\mathrm{S}^{-1}$ of the composite structure and then we apply a numerical method to solve for the total scattered currents. We focus on $S^{-1}$, rather than on $S$, because the former can be written in closed form, whereas determining the latter requires the inversion of $\mathrm{S}^{-1}$ - which can be effected only numerically. 
In order to determine $\mathrm{S}^{-1}$, we consider the $N_{D}$ bricks depicted in Fig. 1. In response to the incident fields $F^{\mathrm{i}}$, scattered currents develop on each brick's boundary. In particular, thanks to linearity, $q_{k}^{\mathrm{s}}$ still follow from (7), provided we include among the incident currents both the contribution from the external sources and the terms due to the presence of all the other $N_{D}-1$ bricks. Symbolically, this reads

$$
q_{k}^{\mathrm{s}}=\mathrm{S}_{k k} q_{k, \text { tot }}^{\mathrm{i}}=\mathrm{S}_{k k}\left(q_{k}^{\mathrm{i}}+\sum_{n \neq k} \mathrm{~T}_{k n} q_{n}^{\mathrm{s}}\right), \quad \forall k
$$

where the summation extends over all but the $k$ th brick. We express the extra contributions by means of (dimensionless) transfer operators $\mathrm{T}_{k n}$, namely

$$
q_{k(n)}^{\mathrm{i}}=\mathrm{T}_{k n} q_{n}^{\mathrm{s}}=\int_{\partial \mathcal{D}_{n}^{-}} \mathrm{d}^{2} r^{\prime} T_{k n}\left(\boldsymbol{r}, \boldsymbol{r}^{\prime}\right) q_{n}^{\mathrm{s}}\left(\boldsymbol{r}^{\prime}\right), \quad k \neq n
$$

which map scattered currents on $\partial \mathcal{D}_{n}^{-}$to incident currents on $\partial \mathcal{D}_{k}^{+}$. We obtain $\mathrm{T}_{k n}$ by first relating $q_{n}^{\mathrm{s}}$ to the tangential incident fields $F_{\mathrm{t} k}^{\mathrm{i}}$ via the relevant propagator, i.e.,

$$
F_{\mathrm{t} k}^{\mathrm{i}}=\mathrm{P}_{k n} q_{n}^{\mathrm{s}}=\int_{\partial \mathcal{D}_{n}^{-}} \mathrm{d}^{2} r^{\prime} P_{k n}\left(\boldsymbol{r}, \boldsymbol{r}^{\prime}\right) q_{n}^{\mathrm{s}}\left(\boldsymbol{r}^{\prime}\right), \quad \boldsymbol{r} \in \partial \mathcal{D}_{k}^{+}
$$

which is also outlined in Table I. Second, we plug $F_{\mathrm{t} k}^{\mathrm{i}}$ from (14) into (4), which we solve for $q_{k(n)}^{\mathrm{i}}$. Thus, we find the following expression for the transfer operators

$$
\mathrm{T}_{k n}=\left(\mathrm{P}_{k k}^{\mathrm{i}}\right)^{-1} \mathrm{P}_{k n}, \quad k \neq n
$$

again in a format well-suited for numerical evaluation.

To complete the derivation of $\mathrm{S}^{-1}$, we solve the $N_{D}$ coupled BIEs implied in (12) with respect to $q_{k}^{\mathrm{i}}$. Upon grouping all of the scattered and incident currents columnwise into $q^{\mathrm{s}}$ and $q^{\mathrm{i}}$, we can express the result succinctly as

$$
\mathrm{S}^{-1} q^{\mathrm{s}}=q^{\mathrm{i}}
$$

with

$$
\begin{aligned}
\mathrm{S}^{-1} & =\left[\begin{array}{cccc}
\mathrm{S}_{11}^{-1} & -\mathrm{T}_{12} & \cdots & -\mathrm{T}_{1 N_{D}} \\
-\mathrm{T}_{21} & \mathrm{~S}_{22}^{-1} & \cdots & -\mathrm{T}_{2 N_{D}} \\
\vdots & \vdots & \ddots & \vdots \\
-\mathrm{T}_{N_{D} 1} & -\mathrm{T}_{N_{D} 2} & \cdots & \mathrm{S}_{N_{D} N_{D}}^{-1}
\end{array}\right] \\
q^{\mathrm{s}} & =\left[q_{1}^{\mathrm{s}}, \cdots, q_{N_{D}}^{\mathrm{s}}\right]^{t}, \quad q^{\mathrm{i}}=\left[q_{1}^{\mathrm{i}}, \cdots, q_{N_{D}}^{\mathrm{i}}\right]^{t} .
\end{aligned}
$$

Equation (16) along with (17) constitutes the formulation of the EM problem represented in Fig. 1. The total inverse scattering operator fully and elegantly incorporates the underlying physics. In fact, all of its entries obviously depend on the shape of the bricks, but only the diagonal entries $\mathrm{S}_{k k}^{-1}$ convey information about the objects comprising the structure [see (11)]. In contrast, the off-diagonal entries, $-\mathrm{T}_{k n}$, (which tell us how the bricks interact) quite remarkably do not depend on the bricks' content, but solely on their relative position in the structure, as is evident from (15). This means that if, for the sake of argument, we allow for a change either in the EM properties or in the shape of the object within each brick, only $\mathrm{S}_{k k}^{-1}$ have to be re-computed-which adds to the efficiency of the LEGO approach.
As in general we cannot carry out the inversion of (17) in closed form-a notable exception being the instance of just two bricks [3] -it is now clear why we stick to $S^{-1}$. On the other hand, the numerical inversion [22], feasible as it can be insofar as the resulting matrix can be handled, is unnecessary, for we can directly solve (16) efficiently with the MoM and the EEM, as we shall discuss in the next section. Besides, once the $q_{k}^{S}$ have been computed, the total incident currents on $\partial \mathcal{D}_{k}^{+}$follow from the rightmost term in (12).

\section{The Eigencurrent Expansion Method}

We now describe how to accomplish the numerical solution of (16) by the MoM [2], [19], combined with the EEM [15].

\section{A. Concept}

Loosely speaking, the very idea behind the EEM is to expand $q^{\mathrm{s}, \mathrm{i}}$ on a set of basis functions $E$ which are "approximations" to the eigenfunctions of $\mathrm{S}^{-1}$, say $\left\{s_{m}^{(k)}\right\}, m \in \mathbb{N}$. We dub $\left\{s_{m}^{(k)}\right\}$ eigencurrents, for in the light of (2) $\mathrm{S}^{-1}$ maps scaled currents to scaled currents. We mention in passing that the EEM, in the form just stated, differs from the one applied in [15], [16, Ch. 5], since there the set $E$ was actually used to start an iterative process aimed at calculating the true eigencurrents of the relevant operator.

To figure out what the set $E$ should be, we neglect for a moment the multiple reflections occurring in the composite structure of Fig. 1; as a consequence, the transfer operators in (17) vanish and we are left with a block diagonal operator. In this ideal case, the eigencurrents $\left\{e_{m}^{(k)}\right\}$ of $S^{-1}$ simply ensue upon juxtaposing the eigencurrents of the individual $S_{k k}$, say $\left\{u_{m}^{(k)}\right\}$. For instance, the generic eigencurrent is defined as

$$
e_{m}^{(k)}=\left[0, \ldots, 0, u_{m}^{(k)}, 0, \ldots\right]^{t}
$$

i.e., it is zero over all $\partial \mathcal{D}_{n}$ except $\partial \mathcal{D}_{k}$.

If we do take into account the interactions among the LEGO bricks, the set $\left\{e_{m}^{(k)}\right\}$ will no longer represent the true eigencurrents of $S^{-1}$. Nevertheless, owing to their definition, we do expect $\left\{e_{m}^{(k)}\right\}$ to be approximations to $\left\{s_{m}^{(k)}\right\}$, therefore we adopt them to form the set $E$ and we still name them eigencurrents. More precisely, we speculate that only few elements of $E$, namely, those associated with the larger eigenvalues of each $\mathrm{S}_{k k}$, will depart considerably from the corresponding $\left\{s_{m}^{(k)}\right\}$ : we say that these eigencurrents are coupled. Conversely, we expect most elements of $E$, viz., those relative to the higher-order eigenvalues, to constitute increasingly better approximations to $\left\{s_{m}^{(k)}\right\}$. The latter may properly be termed uncoupled.

In light of these observations, we stipulate that the entries of the resulting MoM matrix, when obtained by adopting $E$ as a set of basis and test functions, are not equally meaningful. As we will show in Section IV-C, this observation is crucial to reduce the size of the algebraic system drastically without jeopardizing the accuracy of the solution.

\section{B. Algorithm}

Since in reality we do not know the elements of $E$ analytically, we accomplish the numerical implementation of the EEM as follows. 

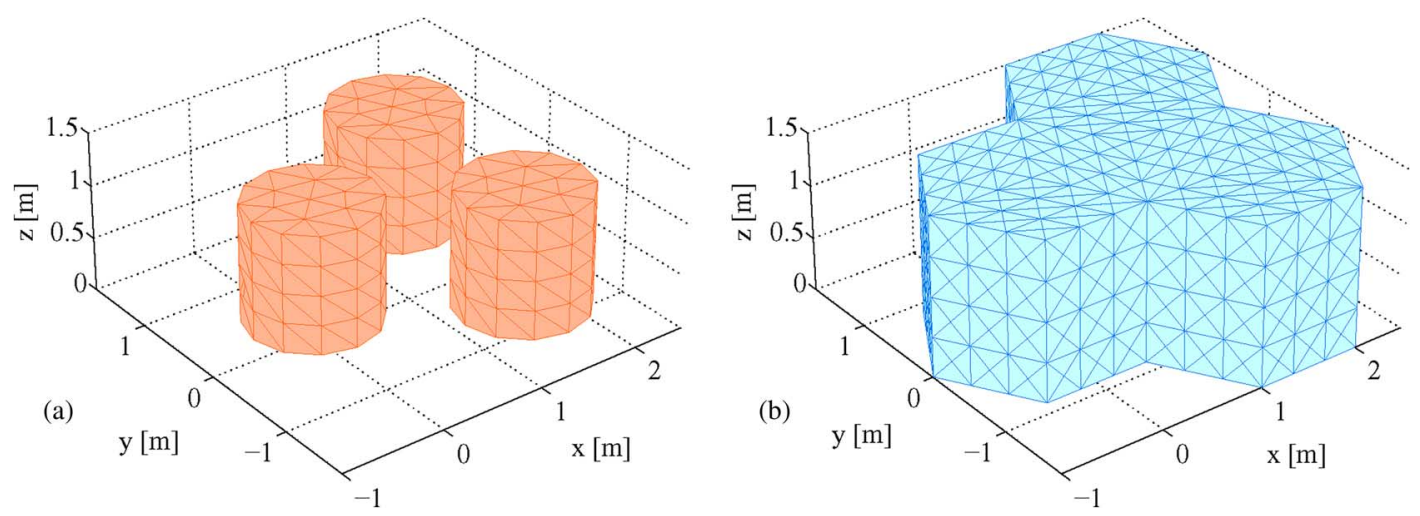

Fig. 3. For LEGO validation: a composite structure made of three dielectric cylinders (a) is represented with three LEGO bricks which are hexagonal prisms (b); both cylinders' and bricks' boundaries are modelled with a 3-D triangular-facet mesh on which RWG functions are defined.

Firstly, we model each boundary $\partial \mathcal{D}_{k}$ by a 3-D triangularfacet mesh, on which we introduce a set $B_{k}$ of $2 N_{F}$ RWG functions [23] to represent the current densities,

$$
q_{k}^{\mathrm{i}}=\sum_{p=1}^{N_{F}}\left[\begin{array}{c}
\boldsymbol{f}_{k p} J_{k p}^{\mathrm{i}} \\
-\boldsymbol{g}_{k p} M_{k p}^{\mathrm{i}}
\end{array}\right], \quad q_{k}^{\mathrm{s}}=\sum_{p=1}^{N_{F}}\left[\begin{array}{c}
\boldsymbol{f}_{k p} J_{k p}^{\mathrm{s}} \\
-\boldsymbol{g}_{k p} M_{k p}^{\mathrm{s}}
\end{array}\right]
$$

where the normalizing intrinsic impedance $\eta$ has been included in the expansion coefficients, and we have chosen $\boldsymbol{f}_{k p}=\boldsymbol{g}_{k p}$, even though conceptually they constitute two distinct elements of $B_{k}$. On $\partial \mathcal{D}_{k}$ we generate a regular mesh (e.g. see Fig. 3(b)) to prevent the facets belonging to different bricks from overlapping awkwardly when two bricks touch one another. This greatly simplifies the calculation of the MoM integrals. Furthermore, we similarly model each object's boundary $\mathcal{S}_{\mathrm{o}}$ and define on it a set $C_{k}$ of $N_{O}$ RWG functions for $q_{\mathrm{o}}$. The exact structure of $C_{k}$ varies depending on the nature of the BIE (8), as outlined in Table II.

Secondly, we apply the MoM to (5), (8), (9), (10) and (14) using the sets $B_{k}$ and $C_{k}$ as basis and test functions along with a symmetric inner product. As a result, we may rewrite (11) and (15) as

$$
\begin{aligned}
& {\left[S_{k k}\right]=-\left[P_{k k}^{\mathrm{s}}\right]^{-1}\left[P_{k \mathrm{o}}\right]\left[X_{\mathrm{oo}}\right]^{-1}\left[P_{\mathrm{o} k}\right]} \\
& {\left[T_{k n}\right]=\left[P_{k k}^{\mathrm{i}}\right]^{-1}\left[P_{k n}\right], \quad k \neq n}
\end{aligned}
$$

where, with evident notation, each matrix represents the algebraic counterpart of the corresponding integral operator. The entries of each matrix in (21) and (22) are double surface integrals involving pairs of RWG functions and can be deduced with the aid of Tables I-III. In particular, when the structure is electrically large, most of the propagation matrices $\left[P_{k n}\right]$ can be evaluated asymptotically as shown in Appendix A.

With these preliminaries, (16), (17) and (18) become

$$
\begin{aligned}
{[S]^{-1}\left[q^{\mathrm{s}}\right] } & =\left[q^{\mathrm{i}}\right] \\
\left([S]^{-1}\right)_{k n} & = \begin{cases}{\left[S_{k k}\right]^{-1}} & k=n, \\
-\left[T_{k n}\right] & k \neq n,\end{cases} \\
\left(\left[q^{\mathrm{s}}\right]\right)_{k} & =\left[q_{k}^{\mathrm{s}}\right], \quad\left(\left[q^{\mathrm{i}}\right]\right)_{k}=\left[q_{k}^{\mathrm{i}}\right]
\end{aligned}
$$

where, e.g., $\left[q_{k}^{\mathrm{s}}\right]$ contains the $2 N_{F}$ expansion coefficients of $q_{k}^{\mathrm{s}}$ and so forth. The total inverse scattering matrix implicitly defined in (24) is of size $2 N_{F} N_{D} \times 2 N_{F} N_{D}$.
Thirdly, for each brick, we compute the $2 N_{F}$ eigenvalues $\lambda_{p k}$ and eigenvectors $v_{p}^{(k)}, p=1, \ldots, 2 N_{F}$, of $\left[S_{k k}\right]$. Note that the spectral decomposition [24] of [ $\left.S_{k k}\right]$ — which may be time consuming - actually has to be effected only once in the particularly meaningful instance when all of the $N_{D}$ LEGO bricks are equal to each other. Next we employ $v_{p}^{(k)}$ to build a larger basis $U$ for spanning the space of $\left[q^{\mathrm{s}, \mathrm{i}}\right]$, namely,

$$
[V]=\operatorname{diag}\left\{\left[V_{k k}\right]\right\}
$$

where $\left[V_{k k}\right]$ stores $v_{p}^{(k)}$ columnwise. The eigenvalues (and accordingly the eigenvectors in $\left[V_{k k}\right]$ ) are arranged in a vector with their real parts in descending order [25]. Note that $U$ is precisely the (finite) algebraic counterpart of the basis $E$, and hence, we term the $2 N_{F} N_{D}$ elements of $U$ eigencurrents.

As a final step, we rephrase (23) in the basis $U$, namely,

$$
[\tilde{S}]^{-1}\left[\tilde{q}^{\mathrm{S}}\right]=\left[\tilde{q}^{\mathrm{i}}\right]
$$

where

$$
\begin{aligned}
& {\left[\tilde{q}^{\mathrm{i}, \mathrm{s}}\right]=[V]^{-1}\left[q^{\mathrm{i}, \mathrm{s}}\right], \quad[\tilde{S}]^{-1}=[V]^{-1}[S]^{-1}[V]} \\
& \left([\tilde{S}]^{-1}\right)_{k n}= \begin{cases}\operatorname{diag}\left\{\lambda_{p k}^{-1}\right\}, & k=n \\
-\left[\tilde{T}_{k n}\right], & k \neq n\end{cases}
\end{aligned}
$$

with

$$
\left[\tilde{T}_{k n}\right]=\left[V_{k k}\right]^{-1}\left[T_{k n}\right]\left[V_{n n}\right] .
$$

The matrix $[\tilde{S}]^{-1}$ would be exactly diagonal if we had neglected the multiple scattering occurring in the structure.

\section{Order Reduction}

Equation (27) is better suited for inversion than (23) is, as the number of entries of $[\tilde{S}]^{-1}$ necessary for solving (16) accurately may be greatly reduced. To this end we first swap rows and columns of $[\tilde{S}]^{-1}$ as follows:

- the entries pertaining to two coupled eigencurrents are moved to the upper left part of the matrix;

- the entries germane to two uncoupled eigencurrents are shifted to the lower right part of the matrix; 
- the entries involving a coupled and an uncoupled eigencurrent are displaced either to the lower left or the upper right corner of the matrix.

In symbols

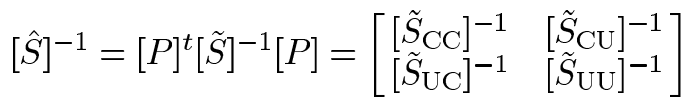

where $[P]$ is a permutation matrix [24]. Based on the coupleduncoupled eigencurrent concept, we now observe that:

- the block matrix $\left[\tilde{S}_{\mathrm{CC}}\right]^{-1}$ is dominant;

- the off-diagonal block matrices $\left[\tilde{S}_{\mathrm{CU}}\right]^{-1},\left[\tilde{S}_{\mathrm{UC}}\right]^{-1}$ must be relatively small and can be neglected altogether;

- the off-diagonal entries of $\left[\tilde{S}_{\mathrm{UU}}\right]^{-1}$ either are null or must be relatively small and can be discarded as well.

As a consequence, we may approximate (31) as

$$
[\hat{S}]^{-1} \approx\left[\begin{array}{cc}
{\left[\tilde{S}_{\mathrm{CC}}\right]^{-1}} & {[0]} \\
{[0]} & {\left[\Lambda_{\mathrm{UU}}\right]^{-1}}
\end{array}\right]
$$

where $\left[\Lambda_{\mathrm{UU}}\right]$ is diagonal and contains all the eigenvalues corresponding to the uncoupled eigencurrents in $U$. At this point, we have turned the problem of solving (16) into the formal inversion of the diagonal matrix $\left[\Lambda_{\mathrm{UU}}\right]^{-1}$ plus the solution of a linear system whose matrix, $\left[\tilde{S}_{\mathrm{CC}}\right]^{-1}$, is far smaller than $[S]^{-1}$ in $(24)$, i.e., if we had used the original RWG functions distributed over all of the $N_{D}$ domains.

Eventually, the expansion coefficients of the scattered currents in the original set $\cup_{k} B_{k}$ may be written as

$$
\left[q^{\mathrm{s}}\right] \approx[V][P]\left[\begin{array}{cc}
{\left[\tilde{S}_{\mathrm{CC}}\right]} & {[0]} \\
{[0]} & {\left[\Lambda_{\mathrm{UU}}\right]}
\end{array}\right][P]^{t}[V]^{-1}\left[q^{\mathrm{i}}\right]
$$

which is the sought for solution to (16) and constitutes the most important result of this work. Furthermore, by also applying the procedure outlined above to the the rightmost part of (12), we may express the total incident currents as

$$
\left[q_{\mathrm{tot}}^{\mathrm{i}}\right] \approx\left[q^{\mathrm{i}}\right]+[V][P]\left[\begin{array}{cc}
{\left[\tilde{T}_{\mathrm{CC}}\right]} & {[0]} \\
{[0]} & {[0]}
\end{array}\right][P]^{t}[V]^{-1}\left[q^{\mathrm{s}}\right]
$$

where $\left[\tilde{T}_{\mathrm{CC}}\right]=\left[\Lambda_{\mathrm{CC}}\right]^{-1}-\left[\tilde{S}_{\mathrm{CC}}\right]^{-1}$, with $\left[\Lambda_{\mathrm{CC}}\right]$ a diagonal matrix containing the eigenvalues associated with the coupled eigencurrents in $U$. The matrix $\left[\tilde{T}_{\mathrm{CC}}\right]$ has null diagonal and depends only on the transfer matrices (30). Apparently, (34) can be interpreted as a statement that only the coupled eigencurrents in $U$ contribute to the multiple reflections occurring among the bricks in the structure. In theory, from (12) we may also deduce an alternative expression for the coefficients of the total incident currents, namely,

$$
\left[q_{\mathrm{tot}}^{\mathrm{i}}\right]=\operatorname{diag}\left\{\left[S_{k k}\right]^{-1}\right\}\left[q^{\mathrm{s}}\right]=[V]^{-1} \operatorname{diag}\left\{\lambda_{p k}^{-1}\right\}[V]\left[q^{\mathrm{s}}\right]
$$

which at a first glance seems convenient, in that we have effected the spectral decomposition of $\left[S_{k k}\right]$ for our needs. As a matter of fact, (35) turns out to be quite unstable as the smallest eigenvalues - possibly close to threshold of numerical noise-lead to inaccurate results when inverted.

Equations (33) and (34) are remarkably efficient in many respects:
1) to build $\left[\tilde{S}_{\mathrm{CC}}\right]^{-1}$ we need to compute $\left[\tilde{T}_{k n}\right]$ and $\left[\tilde{T}_{n k}\right]$ for bricks $k$ and $n$, but we only have to store the entries relative to the coupled eigencurrents;

2) in view of (22) and (30) we may get $\left[\tilde{T}_{k n}\right]$ by solving

$$
\left(\left[P_{k k}^{\mathrm{i}}\right]\left[V_{k k}\right]\right)\left[\tilde{T}_{k n}\right]=\left[P_{k n}\right]\left[V_{n n}\right], \forall k, n, k \neq n ;
$$

3) the main diagonal of $\left[\tilde{S}_{\mathrm{CC}}\right]^{-1}$ contains the reciprocal of $\lambda_{k p}$ associated with the coupled eigenvectors; since the corresponding eigenvalues are the largest ones, their inversion is stable;

4) we do not compute $\left[\tilde{S}_{\mathrm{CC}}\right]$, but rather solve the system by effecting the LU factorization [22] of $\left[\tilde{S}_{\mathrm{CC}}\right]^{-1}$;

5) $\left[\Lambda_{U U}\right]^{-1}$ need not be built nor inverted, since we already know $\lambda_{p k}$ from the spectral decomposition of $\left[S_{k k}\right]$;

6) the action of the permutation matrix $[P]$ is accounted for by swapping columns (rows) of the matrices by which $[P]$ $\left([P]^{t}\right)$ is being right- (left-) multiplied;

7) to calculate $\left[\tilde{q}^{i}\right]$ in (27), we do not build nor invert the (block diagonal) eigencurrent matrix $[V]$ as such, but rather we solve the $N_{D}$ systems

$$
\left(\left[P_{k k}^{\mathrm{i}}\right]\left[V_{k k}\right]\right)\left[\tilde{q}_{k}^{\mathrm{i}}\right]=\left[F_{\mathrm{t} k}^{\mathrm{i}}\right], \quad \forall k
$$

ensuing from the algebraic counterparts of (4) and (6);

8) (33) and (34) can be solved simultaneously for multiple sources and, more importantly, this can also be done at later times, provided we save and then load few relatively small matrices (i.e., the LU-decomposed $\left[\tilde{S}_{\mathrm{CC}}\right]^{-1}, \operatorname{diag}\left\{\lambda_{p k}\right\}$, $\left.\left[V_{k k}\right]\right)$.

Last but not least, since only $N_{C} N_{D}$ eigencurrents out of the total $2 N_{F} N_{D}$ in $U$ are likely to be coupled, the size of the matrix to be actually handled (i.e., $\left[\tilde{S}_{\mathrm{CC}}\right]^{-1}$ ) shrinks to $N_{C} N_{D} \times N_{C} N_{D}$ (as compared to the possibly humongous size $2 N_{F} N_{D} \times 2 N_{F} N_{D}$ of $[S]^{-1}$ ). Accordingly, the computational complexity of the overall LEGO-EEM approach is roughly $O\left(N_{C}^{3} N_{D}^{3}\right)$, which is to be contrasted to $O\left(N_{O}^{3} N_{D}^{3}\right)$, i.e. the number of operations needed to invert the matrix resulting from the direct MoM solution of the same EM problem (assuming the relevant systems are solved by LU factorization). Besides, when the object inside $\mathcal{D}_{k}$ happens to fill most of the brick's volume, a finer mesh may be necessary over $\partial \mathcal{D}_{k}$ for capturing the near field fabric accurately. However, even in the worst case scenario, viz. $2 N_{F} \approx N_{O}$, LEGO-EEM will be convenient, since ordinarily $N_{C} \ll 2 N_{F}$.

Finally, the time required to fill all of the transfer matrices in (22) may be further reduced, on the one hand, by taking advantage of possible (translational) symmetries of the structure (thereby most of the $\left[T_{k n}\right]$ matrices coincide and need not be recomputed) and, on the other hand, by carrying out the calculations in parallel, as evaluating $\left[T_{k n}\right]$ involves just a pair of bricks at a time (see Fig. 1).

\section{NUMERICAL RESULTS}

We have implemented the LEGO approach along with the MoM-EEM solution in a numerical code capable of handling arbitrarily shaped bricks which may incorporate both PEC and penetrable objects. A thorough assessment of the correctness of 


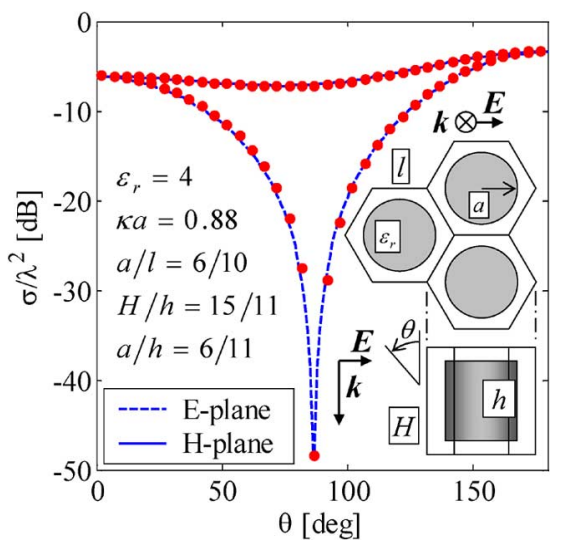

Fig. 4. LEGO validation: the bistatic RCS of the structure in Fig. 3; LEGO solution with $N_{D}=3, N_{C}=10(\bullet)$ versus direct solution with PMCHW BIE $(--/-)$. Inset: cartoon of the LEGO bricks embedding the cylinders (top and side view), and geometrical and physical quantities.

the code and of the overall reliability of the algorithm requires checking, among others:

1) that in the single object instance the physical quantities (e.g. the scattered fields) derivable from the solutions of (7) or (8) do coincide;

2) that in case of a composite structure the MoM-EEM applied to (16) does yield the same results as predicted by the direct solution of the problem tackled with a BIE;

3 ) whether and how fast the EEM solution converges with increasing number of coupled eigencurrents $N_{C} N_{D}$.

The first check above clearly aims at showing the correctness of the numerical calculation of $\left[S_{k k}\right]$. To this end, we have conducted extensive numerical experiments with different shapes of objects and embedding bricks. Our attention has focused on the agreement of the scattered fields in the Fraunhofer region [26], which we derived either directly from the current densities $q_{\mathrm{o}}$ induced over an object's surface or from the scattered current densities $q^{\mathrm{s}}$ distributed over the boundary of the brick embedding the object. The comparisons turned out excellent in all cases. We omit the results for the sake of brevity, since an analogous set of tests involving near fields was carried out in the 2-D instance as reported in [3].

By contrast, we do discuss the validation of the EEM approach, for it constitutes a novelty with respect to the procedure described in [3], and it is a key ingredient of the present 3-D LEGO method. Specifically, to validate the EEM we consider the scattering from the aggregate of three dielectric cylinders in Fig. 3(a), embedded in as many hexagonal LEGO bricks as shown in Fig. 3(b). This structure fits our needs, in that its direct solution with the MoM is available. In our tests, we have computed the bistatic radar cross section (RCS) [27]—in response to a plane wave impinging from above-in two ways:

1) directly, i.e. from $q_{\mathrm{o}}$ calculated through a set of PMCHW equations and the MoM, with $N_{O}=2 \times 216$ RWG functions on each cylinder;

2) with LEGO, from $q^{\mathrm{s}}$, with $N_{D}=3$ hexagonal bricks, ( $N_{O}=2 \times 216,2 N_{F}=2 \times 756$ on each brick), using $N_{C} N_{D}=30$ coupled eigencurrents $\left(N_{C}=10 \ll 2 N_{F}\right)$.
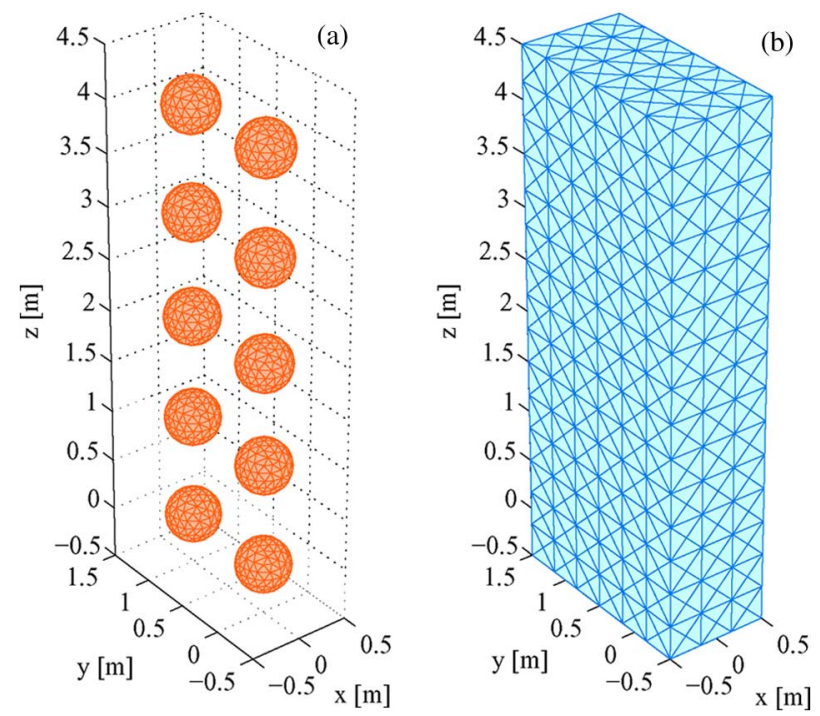

Fig. 5. To study LEGO-EEM convergence: a composite structure made of ten PEC spheres arranged in a rectangular lattice (a) is represented with as many LEGO cubic bricks (b); both spheres' and bricks' boundaries are modelled with a 3-D triangular-facet mesh on which RWG functions are defined.

In Fig. 4 we have plotted the results obtained with the two approaches, in both the E- and H-planes: the RCS's predicted by either method are in excellent agreement, thus confirming the effectiveness of LEGO as well as the validity of the coupled-uncoupled eigencurrent notion outlined in Section IV.

Concerning this, as anticipated, it is also expedient to study the convergence of the solution as a function of the total number of coupled eigencurrents retained to form $\left[\tilde{S}_{\mathrm{CC}}\right]^{-1}$ in (31). To this purpose, we have investigated a more complicated structure, shown in Fig. 5, comprised of ten PEC spheres $\left(\kappa_{0} a=0.733\right.$, $N_{O}=408$ ) arranged in a two-by-five rectangular lattice aligned to the $\hat{z}$-axis. We have embedded the spheres in $N_{D}=10$ cubic bricks $\left(2 N_{F}=648\right)$ and computed the RCS (through $\left.q^{\mathrm{s}}\right)$ for different values of $N_{C}$ : Fig. 6 shows the RCS in the E- (top) and H-plane (bottom).

The RCS obtained with $N_{C}=40(-)$ can be assumed as the reference result, since it is practically indistinguishable from the case $N_{C}=30(\bullet)$; thus, we conclude that in this instance the convergence is reached upon using a total amount of $N_{C} N_{D}=$ 300 coupled eigencurrents. This number should be contrasted to the (virtual) size of the total inverse scattering matrix (24), i.e. $2 N_{F} N_{D}=6480$, in order to appreciate the power of the EEM approach. From Fig. 6 we also notice that the convergence is indeed fast, as there are quite small differences between the cases $N_{C}=20(\boldsymbol{\bullet})$ and $N_{C}=30(\bullet)$. Finally, with just $N_{C}=$ $10(\$)$, even though the agreement with the reference solution is not completely satisfactory as per the RCS levels, nonetheless the trend (as in the predicted number and positions of the nulls) is remarkably good.

The latter numerical experiment on the one hand leads us to conclude that the EEM applied to (23) does converge; on the other, it tells us a posteriori how many eigencurrents are necessary to achieve convergence. In reality, a recipe for picking up the minimum $N_{C}$ a priori would be desirable, but we recognize that a general criterion is quite hard to establish. As a rule of 

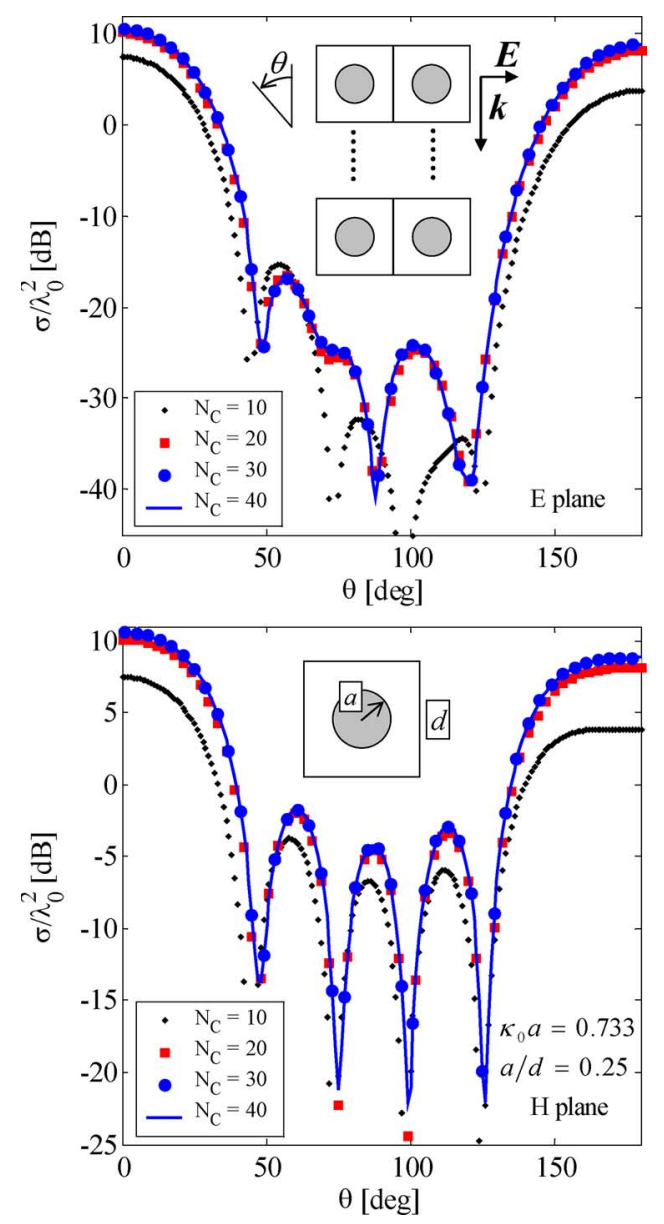

Fig. 6. LEGO-EEM convergence: bistatic RCS of the structure in Fig. 5 in E(top) and $\mathrm{H}$ - (bottom) planes for increasing number $N_{C} N_{D}$ of coupled eigencurrents $\left(N_{D}=10\right)$. Insets: cartoons of the LEGO bricks embedding the spheres, and geometrical and physical quantities.

thumb, $N_{C}$ is mostly affected by the electrical size and distance of the objects in the bricks (based on the established multipole expansion methods [10], [28], one also expects the scattering matrix (29) to become increasingly close to a true diagonal matrix as the bricks are set farther and farther away). Thereby, a sensible statement is that the more electrically close are the objects enclosed in the LEGO bricks, the stronger is the coupling among the nearest ones, and the higher is the number of coupled eigencurrents required for the solution to converge. In this respect, however, since the strongest interactions most certainly take place among adjacent bricks, a preliminary assessment of the EEM convergence for a given problem can be conducted with just few neighboring bricks-which turns out to be a relatively fast procedure and can yield a reliable indication about the minimum $N_{C}$, before facing the solution of the whole large structure.

We note that, as the EEM approach relies on the eigencurrents $v_{p}^{(k)}$ of a single brick (see Section IV-B), the problem of computing them accurately with the MoM deserves attention. This means that in general on a brick's boundary $\partial \mathcal{D}_{k}$ we have to set up a dense enough triangular mesh, say at least ten facets per wavelength, in accordance with a commonly accepted rule [19]. As an exception to this criterion, when LEGO bricks are

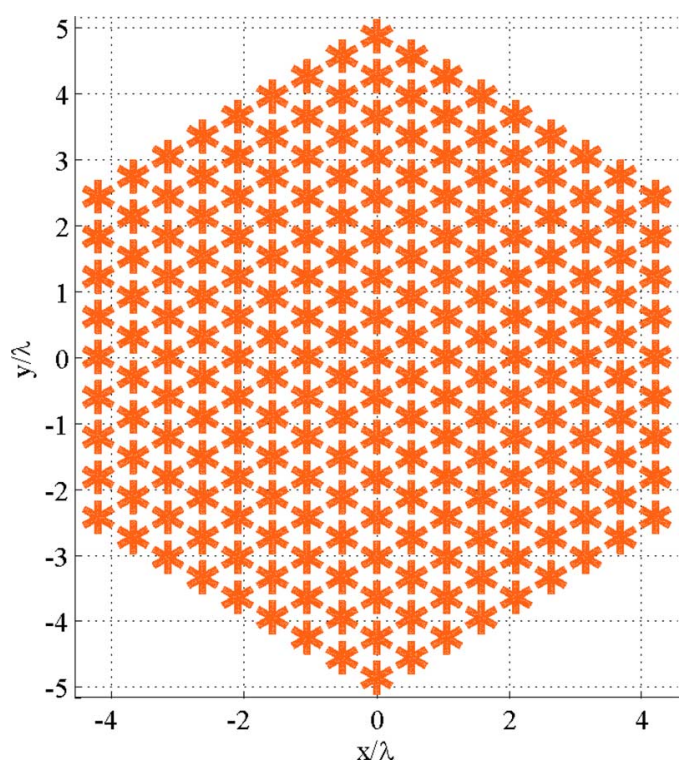

Fig. 7. Case study: 3-D PEC finite-thickness "snowflakes" arranged in a hexagonal lattice for increasing number of elements; the largest configuration $\left(N_{D}=\right.$ 217 ) is shown (see also the insets of Figs. 8 and 9).

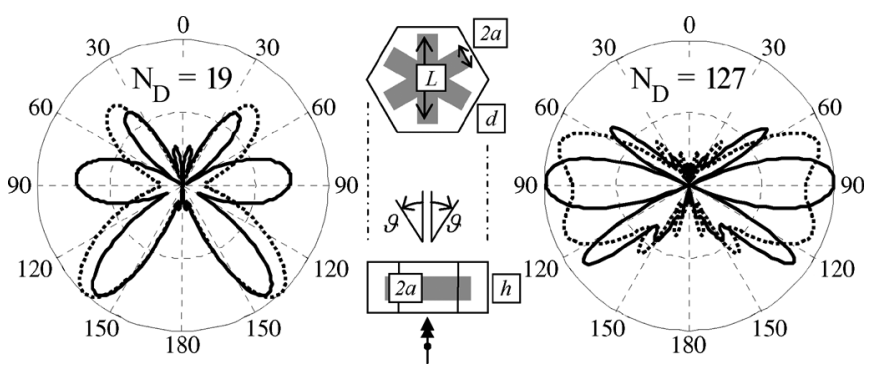

Fig. 8. Radiation pattern (a.u.) of a magnetic dipole in presence of the structure in Fig. 7 for $N_{D}=19$ (left) and $N_{D}=127$ (right): $(-) \phi=0,180^{\circ},(\ldots)$ $\phi=90^{\circ}, 270^{\circ}$. Inset: cartoon of the LEGO brick embedding the "snowflake" (top and side view), geometrical quantities and source.

either relatively far from one another or large as compared to the embedded object, the mesh density on the brick may be coarser than the one on the object without losing accuracy [12, p. 131].

Finally, in order to discuss the time requirements of LEGO, we studied the scattering (in free space) from an aggregate of $N_{D}$ PEC finite-thickness 6-arm "snowflakes" $\left(L=0.5 \lambda_{0}, 2 a=\right.$ $\left.0.1 \lambda_{0}\right)$, arranged in a hexagonal lattice $\left(d=0.35 \lambda_{0}, h=\right.$ $0.2 \lambda_{0}$ ) with an increasingly larger number of concentric rows (from 1 to 8). In Fig. 7 we show the largest configuration (eight rows, $N_{D}=217$ ) we analyzed. Each snowflake is embedded in a regular hexagonal brick modelled with a triangular 3-D mesh, which results in $2 N_{F}=1080$ RWG functions per brick. Then we solved (23) with $N_{C}=10$, with the structure illuminated by a magnetic dipole (i.e., an elemental electric current loop) aligned to the $\hat{z}$-axis and placed thereon at $z_{\mathrm{s}}=$ $-0.125 \lambda_{0}$ below the structure. As an example, the fields radiated by the calculated scattered currents $q^{\mathrm{s}}$ and the magnetic dipole are shown in Fig. 8 for $N_{D} \in\{19,127\}$.

Calculations were carried out on a Linux-based x86_64 workstation equipped with an Intel Xeon $2.66-\mathrm{GHz}$ processor and 8-GB RAM. In Table IV we report, as a function of $N_{D}$, the 
TABLE IV

CPU TIMES vs CHARACTERISTIC SIZES OF THE PROBLEM IN Fig. 7

\begin{tabular}{ccccc}
\hline \hline \multicolumn{5}{c}{$2 N_{F}=1080, N_{C}=10$} \\
\hline$N_{D}$ & $N_{D}\left(N_{D}-1\right)$ & $2 N_{F} N_{D}$ & $N_{C} N_{D}$ & CPU time [s] \\
\hline 7 & 42 & 7560 & 70 & 188 \\
19 & 342 & 20520 & 190 & 287 \\
37 & 1332 & 39960 & 370 & 319 \\
61 & 3660 & 65880 & 610 & 361 \\
91 & 8190 & 98280 & 910 & 607 \\
127 & 16002 & 137160 & 1270 & 940 \\
169 & 28392 & 182520 & 1690 & 1272 \\
217 & 46872 & 234360 & 2170 & 1633 \\
\hline \hline
\end{tabular}

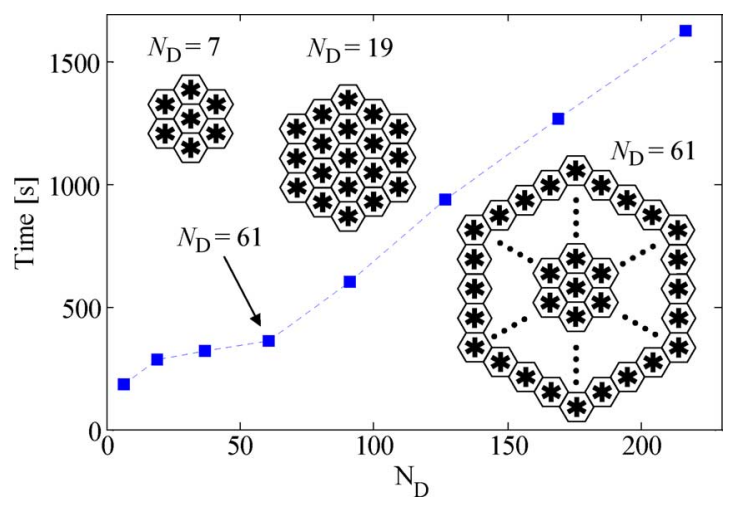

Fig. 9. Overall CPU time as a function of the number of LEGO bricks forming the structure in Fig. 7 (for visualization's sake the square marks are joined with a dashed line). Inset: cartoon of three realizations of the structure with increasing number of snowflakes/bricks.

number $N_{D}\left(N_{D}-1\right)$ of transfer matrices $\left[T_{n k}\right]$ to be computed, the total number $2 N_{F} N_{D}$ of unknowns (which is the size of $[S]^{-1}$ ), the total number $N_{C} N_{D}$ of coupled eigencurrents retained for computation and the overall CPU time. If one compares the size $N_{C} N_{D} \times N_{C} N_{D}$ of $\left[\tilde{S}_{\mathrm{CC}}\right]^{-1}$ to the size $2 N_{F} N_{D} \times 2 N_{F} N_{D}$ of $[S]^{-1}$, then the effectiveness of LEGO in handling large EM problems appears quite evident, and especially so for increasing $N_{D}$. Nevertheless, as the number of transfer matrices $\left[T_{n k}\right]$ grows roughly as $N_{D}^{2}$, one would expect the CPU time to grow quadratically too, but this is not the case. In fact, by taking advantage of the translational symmetries of this structure (see Fig. 7)-which means that we can identify groups of identical transfer matrices-and obtaining the contributions from electrically distant bricks as described in Appendix A, we were able to fill $\left[\tilde{S}_{\mathrm{CC}}\right]^{-1}$ considerably faster than if we had computed all of the $\left[T_{n k}\right]$ independently. As a result, the computational time is seen to grow just linearly with $N_{D}$, as confirmed by the plot in Fig. 9 .

The overall computation time may be deconstructed into various contributions, viz. the time required: 1) to compute the scattering matrix $\left[S_{k k}\right]$ (once for all), 2) to perform its spectral decomposition (once for all), 3) to compute $\left[T_{n k}\right]$ and then fill $\left[\tilde{S}_{\mathrm{CC}}\right]^{-1}$ and 4) to formally invert $\left[\tilde{S}_{\mathrm{CC}}\right]^{-1}$. The first two contributions are fixed and are dominant for relatively small numbers of bricks (up to 37 in our experiment). However, for $N_{D}$ sufficiently large, it is the time needed to fill $\left[\tilde{S}_{\mathrm{CC}}\right]^{-1}$ that becomes dominant: this explains why the onset of the linear rate of increase of CPU time does not occur until $N_{D}=61$.

\section{CONCLUSION}

We have described a novel formulation for 3-D radiation and scattering problems based on the LEGO approach and an integral equation involving the total inverse scattering operator of the structure under study. In the LEGO concept, the objects forming the structure are embedded in EM bricks, each characterized by its own scattering operator. We then solve the relevant equation through the MoM and the EEM, which enables us to address the solution of electrically very large structures (with virtually hundreds of thousands of unknowns) effortlessly and fast. In fact, for a sequence of structures of many identical bricks we have demonstrated that the computation time eventually scales linearly with the number of bricks. On the other hand, although we have presented numerical results in the notable instance when all bricks are equal to one another, the formulation we have proposed is general as per both the shape of the bricks and their contents. In this respect, the local optimization of a large structure can be effected efficiently upon identifying a designated (target) brick - where the EM properties are allowed to vary - and solving once for all the part of the system which does not change. This topic as well as examples of application to real-life devices will be the subject of forthcoming papers.

\section{APPENDIX A \\ ASYMPTOTIC EVALUATION OF $\left[P_{k n}\right]$}

If the spatial extent of the structure spans many wavelengths, then most LEGO EM bricks are likely located in the Fraunhofer region [26] of one another. Under this assumption, the entries of the propagators $\left[P_{k n}\right]$ in (22)-which are double surface integrals stemming from the application of MoM to (15) with due regard to the definition of $\mathrm{P}_{k n}$ in Table I-can be rephrased as combinations of 2-D Fourier transforms of basis and test functions. This significantly speeds up the filling of the matrix.

Specifically, letting $\boldsymbol{f}_{k p}\left(\boldsymbol{g}_{n q}\right)$ be a test (basis) RWG function on $\partial \mathcal{D}_{k}^{+}\left(\partial \mathcal{D}_{n}^{-}\right), \boldsymbol{\tau}$ the position vector of the center of $\mathcal{D}_{k}$ with respect to the center of $\mathcal{D}_{n}, \boldsymbol{\xi}, \boldsymbol{\xi}^{\prime}$ the local position vectors of $f_{k p}, \boldsymbol{g}_{n q}$, respectively, and also $a$ the maximum chord of $\mathcal{D}_{n}$, then for $\tau \gg 2 a^{2} / \lambda$ we have

$$
\begin{aligned}
R & =\left|\boldsymbol{r}-\boldsymbol{r}^{\prime}\right|=\left|\boldsymbol{\xi}-\boldsymbol{\xi}^{\prime}+\boldsymbol{\tau}\right| \approx \tau+\hat{\boldsymbol{\tau}} \cdot\left(\boldsymbol{\xi}-\boldsymbol{\xi}^{\prime}\right) \\
G(R) & \approx G(\tau) \exp \left(-\mathrm{j} \kappa \hat{\boldsymbol{\tau}} \cdot\left(\boldsymbol{\xi}-\boldsymbol{\xi}^{\prime}\right)\right) \\
\nabla_{\mathrm{s}}^{\prime} G(R) & \approx \mathrm{j} \kappa \hat{\boldsymbol{\tau}} G(\tau) \exp \left(-\mathrm{j} \kappa \hat{\boldsymbol{\tau}} \cdot\left(\boldsymbol{\xi}-\boldsymbol{\xi}^{\prime}\right)\right)
\end{aligned}
$$

whence, following the definition of $\mathrm{P}_{k n}$ in Table I and the nomenclature therein, after a little straightforward algebra, the entries of the nonnull blocks of $\left[P_{k n}\right]$ are found to be

$$
\begin{aligned}
\left(\left[P_{k n}\right]_{21}\right)_{p q} \approx-\mathrm{j} \kappa G(\tau) \mathcal{F}_{2}\left\{\boldsymbol{f}_{k p}\right\}_{-\kappa \hat{\boldsymbol{\tau}}} \cdot \hat{\boldsymbol{\tau}} \times \mathcal{F}_{2}\left\{\boldsymbol{g}_{n q}\right\}_{\kappa \hat{\boldsymbol{\tau}}} \\
\left(\left[P_{k n}\right]_{22}\right)_{p q} \approx \mathrm{j} \kappa G(\tau)\left[\mathcal{F}_{2}\left\{\boldsymbol{f}_{k p}\right\}_{-\kappa \hat{\tau}} \cdot \mathcal{F}_{2}\left\{\boldsymbol{g}_{n q}\right\}_{\kappa \hat{\boldsymbol{\tau}}}\right. \\
\left.-\mathcal{F}_{2}\left\{\boldsymbol{f}_{k p}\right\}_{-\kappa \hat{\boldsymbol{\tau}}} \cdot \hat{\boldsymbol{\tau}} \mathcal{F}_{2}\left\{\boldsymbol{g}_{n q}\right\}_{\kappa \hat{\boldsymbol{\tau}}} \cdot \hat{\boldsymbol{\tau}}\right]
\end{aligned}
$$

where, e.g.,

$$
\mathcal{F}_{2}\left\{\boldsymbol{g}_{n q}\right\}_{\kappa \hat{\boldsymbol{\tau}}}=\int \mathrm{d}^{2} \xi^{\prime} \boldsymbol{g}_{n q}\left(\boldsymbol{\xi}^{\prime}\right) \exp \left(\mathrm{j} \kappa \hat{\boldsymbol{\tau}} \cdot \boldsymbol{\xi}^{\prime}\right)
$$

and similarly for $\mathcal{F}_{2}\left\{f_{k p}\right\}_{-\kappa \hat{\tau}}$. Thanks to (39) and (40), when $\tau \gg 2 a^{2} / \lambda$ the process of filling $\left[P_{k n}\right]$ reduces essentially to the closed-form calculation of (at most) $2 N_{F}$ Fourier transforms 
[29, Appendix C] in contrast to the numerical evaluation of $2 N_{F}^{2}$ double surface integrals normally required.

\section{ACKNOWLEDGMENT}

The authors would like to thank the reviewers for their positive comments and useful suggestions which helped refine the manuscript.

\section{REFERENCES}

[1] O. C. Zienkiewicz, The Finite Element Method in Engineering Science. London: McGraw-Hill, 1971.

[2] R. F. Harrington, Field Computation by Moment Methods. New York: MacMillan, 1968.

[3] A. M. van de Water, B. P. de Hon, M. C. van Beurden, A. G. Tijhuis, and P. de Maagt, "Linear embedding via Green's operators: A modeling technique for finite electromagnetic band-gap structures," Phys. Rev. $E$, vol. 72, pp. 1-11, 2005.

[4] A. M. van de Water, B. P. de Hon, A. G. Tijhuis, and M. C. van Beurden, "Electromagnetic embedding," presented at the 29th URSI Gen. Assem., Jul. 2005.

[5] V. Lancellotti, B. P. de Hon, and A. G. Tijhuis, "Electromagnetic modelling of large complex 3-D structures with LEGO and the eigencurrent expansion method," in An. and Prop. Sym., Jun. 2009.

[6] G. Kron, "A set of principles to interconnect the solutions of physical systems," J. Appl. Phys., vol. 24, no. 8, pp. 965-980, 1953.

[7] R. E. Collin, Foundations for Microwave Engineering. New York: McGraw-Hill, 1992.

[8] R. E. Collin, Field Theory of Guided Waves. Piscataway, NJ: IEEE Press, 1991.

[9] R. Orta, R. Tascone, and R. Zich, "A unified formulation for the analysis of general frequency selective surfaces," Electromagnetics, no. 4, pp. 307-329, 1985.

[10] N. Engheta, W. D. Murphy, V. Rokhlin, and M. S. Vassiliou, "The fast multipole method (FMM) for electromagnetic problems," IEEE Trans. Antennas Propag., vol. 40, pp. 634-641, Jun. 1992.

[11] W. C. Chew and C. Lu, "The use of Huygens' equivalence principle for solving the volume integral equation of scattering," IEEE Trans. Antennas Propag., vol. 41, pp. 897-904, Jul. 1993.

[12] M.-K. Li and W. C. Chew, "Wave-field interaction with complex structures using equivalence principle algorithm," IEEE Trans. Antennas Propag., vol. 55, pp. 130-138, Jan. 2007.

[13] I. Lindell, "Huygens' principle in electromagnetics," Proc. Inst. Elect. Eng. Sci., Meas. Technol., vol. 143, pp. 103-105, Mar. 1996.

[14] A. M. van de Water, "LEGO: Linear Embedding via Green's Operators," Ph.D. dissertation, Technische Univ. Eindhoven, The Netherlands, 2007.

[15] D. Bekers, "Finite Antenna Arrays, an Eigencurrent Approach," Ph.D. dissertation, Technische Univ. Eindhoven, The Netherlands, 2004.

[16] G. Addamo, "Electromagnetic Waves in Loaded Cylindrical Structures: A Radial Transmission Line Approach," Ph.D. dissertation, Technische Univ. Eindhoven, The Netherlands, 2008.

[17] A. E. H. Love, "The integration of the equations of propagation of electric waves," Philos. Trans. R. Soc. London, ser. A, vol. 197, pp. 1-45, 1901.

[18] R. F. Harrington, Time-Harmonic Electromagnetic Fields. London: McGraw-Hill, 1961.

[19] A. F. Peterson, S. L. Ray, and R. Mittra, Computational Methods for Electromagnetics. Piscataway, NJ: IEEE Press, 1998.

[20] P. Yla-Oijala and M. Taskinen, "Calculation of CFIE impedance matrix elements with RWG and $\mathbf{n} \times$ RWG functions," IEEE Trans. Antennas Propag., vol. 51, pp. 1837-1846, Aug. 2003.

[21] P. Yla-Oijala and M. Taskinen, "Well-conditioned muller formulation for electromagnetic scattering by dielectric objects," IEEE Trans. Antennas Propag., vol. 53, pp. 3316-3323, Oct. 2005.

[22] D. Bau, III and L. N. Trefethen, Numerical Linear Algebra. Philadelphia, PA: Soci. Indus. Ap. Math., 1997.

[23] S. M. Rao, D. R. Wilton, and A. W. Glisson, "Electromagnetic scattering by surfaces of arbitrary shape," IEEE Trans. Antennas Propag., vol. 30, pp. 409-418, 1982.

[24] G. H. Golub and C. F. V. Loan, Matrix Computations. Baltimore, MD: Johns Hopkins Univ. Press, 1996.

[25] E. Anderson, Z. Bai, C. Bischof, S. Blackford, J. Demmel, J. Dongarra, J. D. Croz, A. Greenbaum, S. Hammarling, A. McKenney, and D. Sorensen, LAPACK Users' Guide. Philadelphia, PA: SIAM, 1999.

[26] C. A. Balanis, Antenna Theory: Analysis and Design. New York: Wiley, 1997

[27] C. A. Balanis, Advanced Engineering Electromagnetics. New York: Wiley, 1989.
[28] J. D. Jackson, Classical Electrodynamics, 3rd ed. Chichester, U.K. Wiley, 1999.

[29] V. Lancellotti, D. Milanesio, R. Maggiora, G. Vecchi, and V. Kyrytsya, "TOPICA: An accurate and efficient numerical tool for analysis and design of ICRF antennas," Nuclear Fusion, vol. 46, no. 7, pp. S476-S499, 2006.

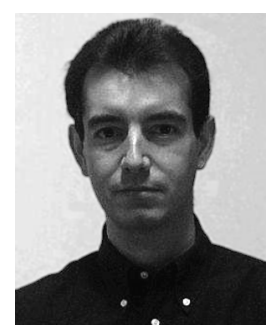

Vito Lancellotti (M'04) was born in Torino, Italy, in 1968. He received the Laurea (M.Sc.) degree (with honors) in electrical engineering and the Ph.D. degree in electronics and communications both from Politecnico di Torino, Italy, in 1995 and 1999, respectively.

In early 1999, he joined Telecom Italia Lab, Torino, as a Senior Researcher and was involved in projects concerning TCP/IP and ATM networks. In June 2000, he became Senior Researcher at the Milan-based subsidiary of Corning (now Avanex), where he worked on broadband electro-optic lithium niobate modulators and optical waveguides. From 2002 to 2008, he served as a Research Fellow and Lecturer in the Department of Electrical Engineering, Politecnico di Torino, where he substantially contributed to the development of the TOPICA code, devised for analysis and design of plasma facing antennas for magnetically controlled nuclear fusion. In 2005, he was appointed Visiting Scientist at Massachusetts Institute of Technology, Cambridge, and in 2007 conducted research at the Max-Planck-Institut fur Plasmaphysik, Garching (Germany). As of April 2008, he is a Research Fellow with the Faculty of Electrical Engineering of Eindhoven University of Technology, Eindhoven, The Netherlands. His current research interests mainly concern efficient techniques for the computational modeling of electromagnetic fields in large structures.

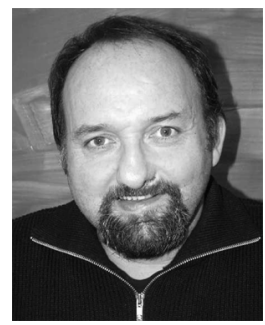

Bastiaan P. de Hon was born in Amstelveen, The Netherlands, in 1966. He received the M.Sc. and Ph.D. degrees (both cum laude) in electrical engineering from the Delft University of Technology, Delft, The Netherlands, in 1991 and 1996, respectively.

Since 1996, he has been with the Electromagnetics Group, Department of Electrical Engineering, Eindhoven University, Eindhoven, The Netherlands, from 1996 until 2000, on a fellowship awarded by the Royal Netherlands Academy of Arts and Sciences, and from 2000 as a Lecturer. He has been a summer student at CERN, Geneva (Switzerland) and at Schlumberger Cambridge Research, Cambridge, U.K., and a Visiting Scientist at the University of Tel Aviv, Tel Aviv, Israel, and the University of Glasgow, U.K. His research interests include theoretical and numerical aspects of electromagnetic, acoustic and elastic wave phenomena.

Dr. de Hon received the Steven Hoogendijk Award for his Ph.D. dissertation in 1998.

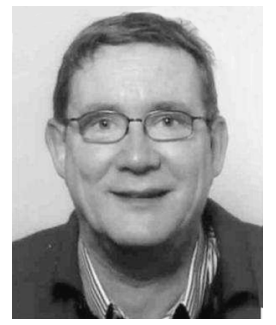

Anton G. Tijhuis (M'88) was born in Oosterhout N.B., The Netherlands, in 1952. He received the M.Sc. degree in theoretical physics from Utrecht University, Utrecht, The Netherlands, in 1976, and the Ph.D. degree (cum laude) from the Delft University of Technology, Delft, The Netherlands, in 1987.

From 1976 to 1986 and 1986 to 1993, he was an Assistant and Associate Professor with the Laboratory of Electromagnetic Research, Faculty of Electrical Engineering, Delft University of Technology. In 1993, he became a Full Professor of electromagnetics with the Faculty of Electrical Engineering, Eindhoven University of Technology, Eindhoven, The Netherlands. He has been a Visiting Scientist with the University of Colorado at Boulder, the University of Granada, Granada, Spain, the University of Tel Aviv, Tel Aviv, Israel, and with McDonnell Douglas Research Laboratories, St. Louis, MO. Since 1996, he has been a Consultant with TNO Defence, Security, and Safety, The Hague, The Netherlands. His research interests are the analytical, numerical, and physical aspects of the theory of electromagnetic waves. In particular, he is involved with efficient techniques for the computational modeling of electromagnetic fields and their application to detection and synthesis problems from several areas of electrical engineering. 